\title{
Open
}

\section{Transcutaneous immunization as preventative and therapeutic regimens to protect against experimental otitis media due to nontypeable Haemophilus influenzae}

\author{
LA Novotny ${ }^{1}$, JD Clements ${ }^{2}$ and LO Bakaletz ${ }^{1,3}$
}

\begin{abstract}
We have developed three nontypeable Haemophilus influenzae (NTHI) adhesin-derived immunogens that are significantly efficacious against experimental otitis media (OM) due to NTHI when delivered parenterally. We now expanded our preventative immunization strategies to include transcutaneous immunization (TCl) as a less invasive, but potentially equally efficacious, regimen to prevent OM due to NTHI. Additionally, we examined the potential of $\mathrm{TCl}$ as a therapeutic immunization regimen to resolve ongoing experimental OM. Preventative immunization with NTHI outer membrane protein (OMP) P5- and type IV pilus-targeted immunogens, delivered with the adjuvant LT(R192G-L211A), induced significantly earlier clearance of NTHI from the nasopharynges and middle ears of challenged chinchillas compared with receipt of immunogen or adjuvant alone. Moreover, therapeutic immunization resulted in significant resolution of established NTHI biofilms from the middle ear space of animals compared with controls. These data advocate $\mathrm{TCl}$ with the adhesin-directed immunogens as an efficacious regimen for prevention and resolution of experimental NTHI-induced OM.
\end{abstract}

\section{INTRODUCTION}

Nontypeable Haemophilus influenzae (NTHI) is a predominant bacterial agent of the prevalent pediatric disease otitis media $(\mathrm{OM})$, and is also responsible for multiple diseases of the upper and lower respiratory tracts of both children and adults. ${ }^{1}$ The economic burden of NTHI-induced diseases, including OM, is significant because of the treatment and surgical management costs. ${ }^{2}$ Complications of OM, for example, hearing loss, are associated with behavioral, educational, and language development delays of this very young population. ${ }^{3}$ With the goal to prevent NTHI-induced OM, many research efforts have focused on the development of vaccines that target outer membrane proteins (OMPs), other surface proteins, and lipooligosaccharide expressed by this bacterium. ${ }^{4}$

Our lab has concentrated on two of the multiple adhesins expressed by NTHI: OMP P5 and the type IV pilus (Tfp). Specifically, we have designed three vaccine candidates: a 40-mer synthetic chimeric peptide immunogen called "LB1" that incorporates a 19-mer B-cell epitope from OMP P5 that has been colinearly synthesized with a T-cell promiscuous epitope from measles virus fusion protein; ${ }^{5}$ a recombinant protein called "rsPilA" that represents a mature, N-terminally truncated, and soluble PilA subunit protein of the Tfp; ${ }^{6}$ and a chimeric immunogen called "chimV4" in which modified rsPilA serves as both immunogen and carrier molecule for a 24-mer epitope of OMP P5 that is positioned at its $\mathrm{N}$-terminus. ${ }^{6}$ Antibody induced by parenteral immunization with any of these immunogens confers significant protection against NTHIinduced OM in a chinchilla model of viral-bacterial superinfection. ${ }^{6,7}$ We now wanted to expand our vaccine delivery strategies to develop a noninvasive but potentially equally efficacious method, transcutaneous immunization (TCI).

TCI, the application of a vaccine onto intact skin, induces an immune response by engaging antigen-presenting cells

\footnotetext{
${ }^{1}$ The Research Institute at Nationwide Children's Hospital, Department of Pediatrics, Center for Microbial Pathogenesis and The Ohio State University College of Medicine, Columbus, Ohio, USA. ${ }^{2}$ Department of Microbiology and Immunology, Tulane University Health Sciences Center, New Orleans, Louisiana, USA. ${ }^{3}$ Nationwide Children's Hospital, Department of Otolaryngology and The Ohio State University College of Medicine, Columbus, Ohio, USA. Correspondence: LO Bakaletz (Lauren.Bakaletz@NationwideChildrens.org)

Received 30 June 2010; accepted 18 January 2011; published online 16 February 2011. doi:10.1038/mi.2011.6
} 
present within the epidermis and dermis, the Langerhan's cells and dermal dendritic cells (DCs), respectively. ${ }^{8}$ There are multiple benefits to this immunization route, which include the simplicity and noninvasive nature of delivery, reduced cost as syringes, needles, and trained medical professionals are not required to deliver the vaccine, and the prospect for greater vaccine distribution beyond developed countries because of typically cheaper production costs. ${ }^{9}$ Previous studies show that TCI with bacterial or viral proteins and other peptide antigens induces an immune response in both animals and humans. ${ }^{10-12}$ Furthermore, via use of animal models, there is evidence of protection against subsequent bacterial, viral, or toxin challenge. ${ }^{13-16}$ Whereas parenteral immunization elicits primarily a systemic immune response, TCI induces both systemic and mucosal immunity. ${ }^{17} \mathrm{OM}$ is a disease of the uppermost respiratory tract, and therefore the ability to induce immunity at the mucosae of this anatomical region has the potential to reduce, or preferably prevent, the onset of disease in the middle ear.

NTHI-induced diseases of the respiratory tract, including $\mathrm{OM}$, can be chronic and/or recurrent in nature, a consequence of biofilms present on the respiratory mucosae. ${ }^{18}$ Specific to OM, NTHI biofilms are shown on the middle ear mucosa of children ${ }^{19}$ and upon gross examination of the middle ear in animal models. ${ }^{20}$ These bacterial communities are recalcitrant to antibiotic treatment and resist immune-mediated clearance. Among other NTHI proteins, OMP P5 and Tfp are identified as components of the biofilm matrix. ${ }^{21,22}$ We therefore hypothesized that immunization with NTHI OMP P5- and Tfp-directed immunogens could serve to target each element in vivo, and thus facilitate resolution of OM. Herein, we utilized a chinchilla model of experimental NTHI-induced OM to examine the immune response elicited by TCI with NTHI OMP P5- and Tfp-directed immunogens when delivered with a potent adjuvant, a double mutant of $E$. coli heat-labile enterotoxin (dmLT). ${ }^{16}$ Moreover, we examined the efficacy of TCI when utilized in both preventative and therapeutic immunization strategies to determine the potential of this noninvasive approach to protect against as well as resolve experimental NTHI-induced OM.

\section{RESULTS}

\section{Histological analysis of chinchilla pinna}

Pinnae from naive chinchillas were collected to examine the histological organization of this tissue. A cross-section of a pinna is shown in Figure 1, wherein the epidermis and dermis, supported by elastic connective tissue, were discernible on each side of hyaline cartilage that runs central through the pinna. Similar to human thin skin, the pinna contained hair follicles and sebaceous glands. An abundant stratum corneum was visible as the outermost layer of the epidermis. Therefore, hydration of this keratinized epithelial layer was deemed necessary to enhance the permeability of the skin and thus facilitate both entry of topically applied molecules and sampling by cutaneous antigen-presenting cells, as has been reported. ${ }^{23}$

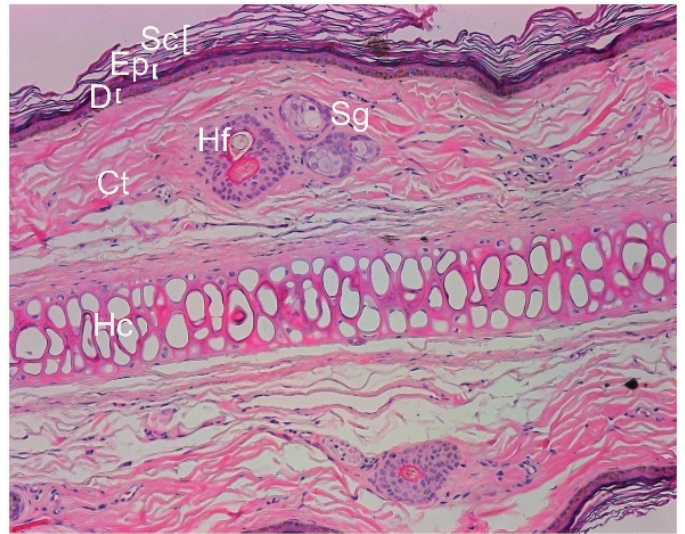

Figure 1 Cross-section through a naive chinchilla pinna stained with hematoxylin and eosin. Chinchilla pinna exhibited characteristics of thin skin and thus was amenable to transcutaneous immunization (TCl) upon hydration of the stratum corneum. Image captured at original magnification $\times 10$. Ct, connective tissue, D, dermis; Ep, epidermis; $\mathrm{Hc}$, hyaline cartilage; $\mathrm{Hf}$, hair follicle and associated sebaceous gland; $\mathrm{Sc}$, stratum corneum; Sg, sebaceous gland.

\section{TCI before NTHI challenge: resolution of nasopharyngeal colonization}

To examine the ability of TCI with NTHI OMP P5-directed candidate "LB1," the Tfp-directed candidate "rsPilA," and the chimeric OMP P5-plus Tfp-directed immunogen "chimV4," to induce clearance of NTHI from the nasopharynges (NP) of challenged chinchillas, periodic NP lavages were performed and the recovered fluids cultured to semiquantitate the relative bacterial load at this anatomical site. Cohorts administered any of the three immunogens without adjuvant and the cohort immunized with dmLT alone had $10^{4}-5 \times 10^{6}$ colony-forming unit (CFU) NTHI in NP lavage fluids beginning 3 days after challenge, and maintained this bacterial load for the remainder of the study period (Figure 2a). In contrast, cohorts that received rsPilA, LB1, or chimV4 admixed with dmLT demonstrated 2- to 4-log fewer NTHI within NP lavage fluids on each of days 7, 10, and 14 after bacterial challenge, a statistically significant result at each time point, compared with cohorts that received respective immunogen only or dmLT only $(P<0.05)$. Moreover, only the three cohorts that received immunogen plus dmLT eliminated NTHI from the NP within 10 or 14 days after challenge.

As an additional assessment of efficacy, the relative percentage of animals with culture-positive NP lavage fluids was calculated for each cohort. At 3 days after NTHI challenge, $100 \%$ of the animals administered immunogen alone or dmLT alone had culture-positive NP lavage fluids, and at least 5/10 (50\%) animals in each of these four cohorts remained culture positive for the entire 14-day study period (Figure 2b). In contrast, a 20-40\% reduction in culture-positive NP lavage fluids was observed 3 days after NTHI challenge in cohorts that received any of the three tested immunogens plus dmLT. Furthermore, at 7 days after NTHI challenge, only $1 / 10(10 \%)$ animals in the cohorts that received $\mathrm{LB} 1+\mathrm{dmLT}$ or rsPilA $+\mathrm{dmLT}$ and $4 / 10(40 \%)$ animals within the cohort administered chimV $4+\mathrm{dmLT}$ had culture-positive NP lavage fluids, and by 14 days after bacterial 

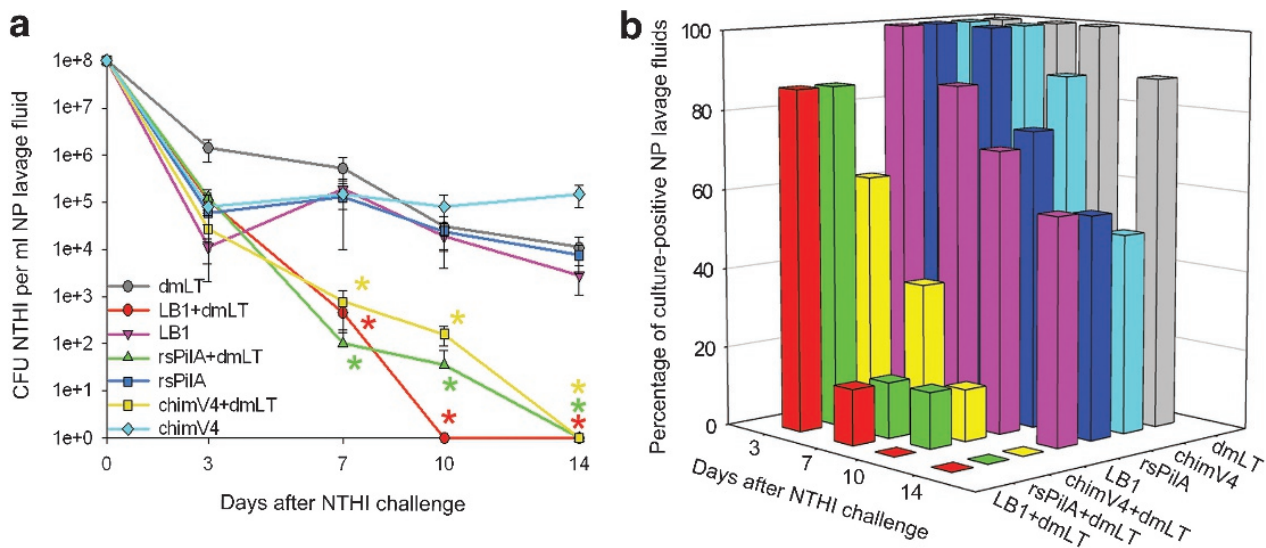

Figure 2 Transcutaneous immunization (TCl) via a preventative regimen induced clearance of nontypeable Haemophilus influenzae (NTHI) from the nasopharynges (NP) of chinchillas. (a) Colonization kinetics that demonstrated that receipt of rsPilA $+\mathrm{dmLT}$, LB1 $+\mathrm{dmLT}$, or chimV4 $+\mathrm{dmLT}$ resulted in rapid clearance of NTHI from the NP relative to the cohorts administered rsPilA, LB1, chimV4, or dmLT alone. *Statistically significant compared with receipt of respective immunogen-only cohort and dmLT-alone cohort $(P<0.05)$. CFU, colony-forming unit; dmLT, double mutant of $E$. coli heatlabile enterotoxin; rsPilA, recombinant soluble PilA. (b) Percentage of colonized NP per cohort showing a reduction in colonization after receipt of $\mathrm{LB} 1+d m L T$, rsPilA +dmLT, or chimV4+dmLT compared with the cohorts administered rsPilA, LB1, chimV4, or dmLT alone.
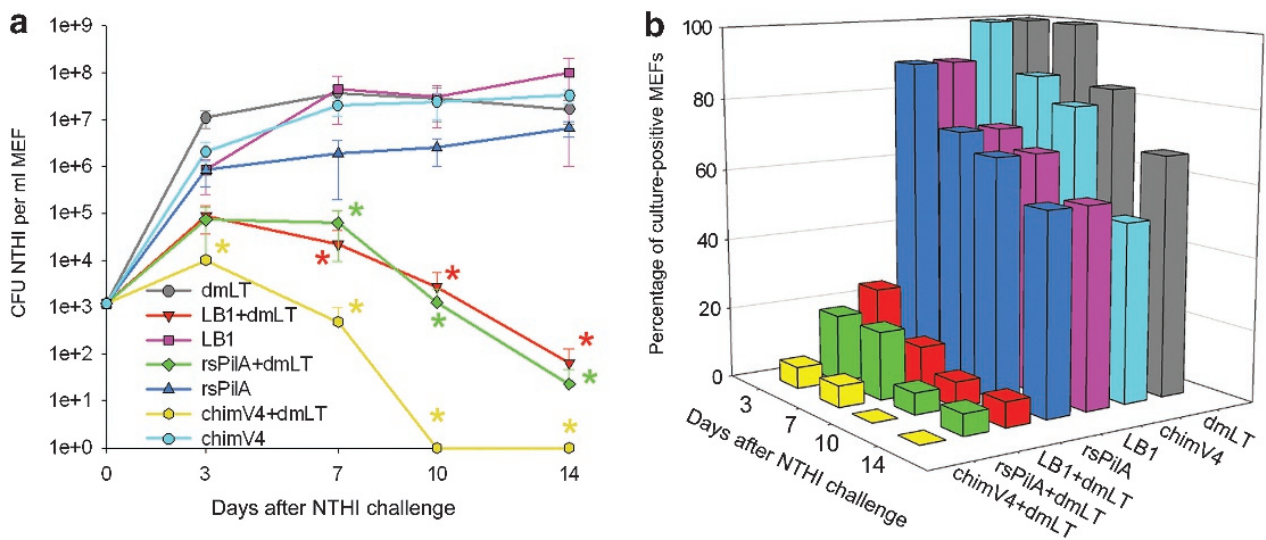

Figure 3 Resolution of otitis media (OM) in directly challenged middle ears after immunization via a preventative regimen. (a) Immunization with rsPilA $+d m L T$, LB1 $+d m L T$, or chimV4 $+d m L T$ induced clearance of nontypeable Haemophilus influenzae (NTHI) from middle ears of chinchillas compared with the cohorts that received rsPilA, LB1, or chimV4, or the cohort that was immunized with dmLT alone. *Statistically significant compared with receipt of respective immunogen-only cohort or dmLT-alone cohort $(P<0.05)$. CFU, colony-forming unit; dmLT, double mutant of $E$. coli heat-labile enterotoxin; rsPilA, recombinant soluble PilA. (b) Reduction in the percentage of culture-positive middle ears per cohort after receipt of rsPilA + dmLT, $\mathrm{LB} 1+\mathrm{dmLT}$, or chimV4 $+\mathrm{dmLT}$ relative to the cohorts that received immunogen or dmLT alone.

challenge all of these latter cohorts had eliminated NTHI from the NP. These data therefore demonstrated that TCI with OMP P5- and Tfp-directed candidates, when delivered in combination with dmLT, resulted in an effective immune response that reduced, and then completely eliminated, NTHI from the NP.

\section{TCI before NTHI challenge: resolution of experimental OM}

As a more rigorous assessment of the protection afforded by TCI, the same chinchillas as challenged intranasally above were also challenged by direct inoculation of the middle ears with 1,000 CFU NTHI to initiate active OM. Middle ear fluids (MEFs), when present as observed by video otoscopy and confirmed by tympanometry, were collected and cultured to determine the relative concentration of NTHI within these fluids. Within 3 days after challenge, the inoculum of 1,000 CFU NTHI had multiplied to $1.1 \times 10^{7} \mathrm{CFU}$ NTHI in MEFs retrieved from animals that received dmLT alone (Figure 3a). As $10^{8} \mathrm{CFU}$
NTHI is typical for sham-immunized chinchillas (alum or monophosphoryl lipid A), ${ }^{24}$ the observed reduction after receipt of dmLT suggested that this adjuvant induced a nonspecific protective effect. Animals administered LB1, rsPilA, or chimV4 alone also exhibited an increase in bacterial concentration to $8.4 \times 10^{5}-2.1 \times 10^{6} \mathrm{CFU}$ NTHI at this time point. Compared with receipt of adjuvant alone, fewer NTHI were detected, which demonstrated that delivery of any of the three immunogens that target NTHI OMP P5 or Tfp induced an enhanced immune response. In contrast, at 3 days after direct challenge, an increase from $1,000 \mathrm{CFU}$ to approximately $8 \times 10^{3}-8 \times 10^{4} \mathrm{CFU}$ NTHI was observed in MEFs collected from animals immunized with any of the three immunogens delivered with dmLT. Moreover, whereas approximately $10^{6}$ to $10^{7} \mathrm{CFU}$ NTHI was detected in MEFs collected from animals immunized with only one of three immunogens or with dmLT alone between 7 and 14 days after challenge, significantly fewer NTHI (approximately 2- to 7-logs 
less) were observed in MEFs collected from animals immunized with LB1, rsPilA, or chimV4 that had been admixed with dmLT $(P<0.05)$. Notably, animals immunized with chimV $4+\mathrm{dmLT}$ resolved $\mathrm{OM}$ within 10 days after challenge.

In terms of the percentage of animals with culture-positive MEFs, similar to that observed in NP lavage fluids, the receipt of rsPilA, LB1, chimV4, or dmLT alone resulted in a minimum of 10/20 ears (50\%) with culture-positive MEFs for the entire 14-day study period in all four cohorts (Figure 3b). However, at 3 days after direct challenge of the middle ear, between 80 and $90 \%$ of middle ears from animals immunized with any of the three immunogens plus dmLT had resolved OM. At 10 days after challenge, only $1 / 20(5 \%)$ middle ears in each cohort administered LB1 or rsPilA plus dmLT remained culture positive for NTHI, and those that received chimV4 $+\mathrm{dmLT}$ had eliminated the bacterium from the middle ear. Therefore, these data demonstrated that TCI with the NTHI OMP P5- and Tfp-directed candidates delivered with dmLT was efficacious and induced elimination of NTHI from the MEFs of challenged animals as well as rapid resolution of experimental OM.

\section{TCI before NTHI challenge: analysis of antibody in serum}

To begin to identify the mechanism(s) for the protection observed, we first examined the relative quantity of immunogen-specific antibody produced systemically. TCI with any of the three immunogens alone induced a fourfold increase in the serum antibody geometric mean titer (GMT) of immunogenspecific $\operatorname{IgG}$ and a two- to four-fold increase in specific $\operatorname{IgA}$ (Table 1) after receipt of all immunizing doses, compared with respective preimmune serum. Inclusion of dmLT in each formulation significantly enhanced the resultant GMT $(P<0.05)$, as a 16- to 64-fold increase in immunogen-specific IgG and a 8 - to 16 -fold increase in specific IgA was detected relative to preimmune serum. Based on the ability to eradicate NTHI from the NP or middle ear, achieving and maintaining a GMT value of at least 160 for immunogen-specific IgG was associated with most rapid clearance observed here.

\section{Recognition of native proteins expressed by NTHI by antibody present within NP lavage fluids induced by a preventative immunization regimen}

Whereas TCI with any of the three candidates induced the production of antibody in serum that recognized the immunogen delivered when assayed by enzyme-linked immunosorbent assay, we wanted to determine if antibody produced mucosally, at the site of NTHI colonization, would bind to native structures expressed by viable NTHI, as these structural proteins would be the target for the antibodies during disease. By flow cytometry, $3.5,3.7$, or $1.0 \%$ more live, unfixed NTHI were positively labeled by antibody in NP lavage fluids collected after TCI with LB1, rsPilA, or chimV4 alone, respectively, relative to receipt of dmLT alone (Table 2). Incubation of NTHI with immune NP lavage fluids collected from animals immunized with LB1, rsPilA, or chimV4 plus dmLT, however, demonstrated an increase of $12.6,11.7$, or $10.0 \%$ in positive labeling of NTHI, respectively, compared with dmLT alone. Relative to fluids collected from
Table 1 Geometric mean titers of immunogen-specific IgG and IgA in serum after $\mathrm{TCl}$ in a preventative immunization regimen

\begin{tabular}{|c|c|c|c|c|}
\hline \multirow[b]{3}{*}{ Cohort } & \multicolumn{4}{|c|}{ Immunogen-specific antibody in serum } \\
\hline & \multicolumn{2}{|c|}{$\lg G$} & \multicolumn{2}{|c|}{$\lg A$} \\
\hline & Pre & Immune & Pre & Immune \\
\hline LB1 & 10 & 40 & 10 & 40 \\
\hline $\mathrm{LB} 1+\mathrm{dmLT}$ & 10 & $320^{*}$ & 10 & $160^{*}$ \\
\hline rsPilA & 10 & 40 & 10 & 40 \\
\hline rsPilA + dmLT & 10 & $160^{*}$ & 10 & 80 \\
\hline chimV4 & 10 & 40 & 10 & 20 \\
\hline chimV4 + dmLT & 10 & $640^{*}$ & 10 & $160^{*}$ \\
\hline
\end{tabular}

Abbreviations: $\mathrm{dmLT}$, double mutant of $E$. coli heat-labile enterotoxin; Ig. immunoglobulin; rsPilA, recombinant soluble PilA; TCI, transcutaneous immunization. ${ }^{\star} P<0.05$ compared with respective immunogen-only titer.

Table 2 Recognition of native proteins expressed by NTHI by antibody in NP lavage fluids as assessed by flow cytometry

\begin{tabular}{lc}
\hline Cohort & $\begin{array}{c}\text { Percentage of labeled NTHI } \\
\text { (relative to labeling by serum from } \\
\text { animals that received dmLT) (\%) }\end{array}$ \\
\hline LB1 & 3.5 \\
LB1 + dmLT & 12.6 \\
rsPilA & \\
rsPilA + dmLT & 3.7 \\
& 11.7 \\
chimV4 & \\
chimV4 + dmLT & 1.0 \\
\hline
\end{tabular}

Abbreviations: $\mathrm{dmLT}$, double mutant of $E$. coli heat-labile enterotoxin; NP, nasopharynge; NTHI, nontypeable Haemophilus influenzae; rsPilA, recombinant soluble PilA

animals that received only immunogen, a 3.2- to 10 -fold greater labeling of NTHI was achieved when NP lavage fluids from immune animals were assayed, which suggested a greater relative antibody titer and/or greater affinity of the antibody for its target was present within these mucosal secretions. Thus, TCI with the synthetic peptide or recombinant protein immunogens plus dmLT induced the production of mucosal antibody that recognized native structures expressed by NTHI that likely contributed to the rapid bacterial clearance observed.

Resolution of established NTHI biomass in the middle ear via use of a therapeutic immunization regimen

To examine the therapeutic potential of TCI with the NTHI OMP P5- and Tfp-directed immunogens, we now first challenged chinchillas by direct inoculation of the middle ear with NTHI strain 86-028NP and allowed a robust biofilm to form in the middle ear space. Previous work has established that when 


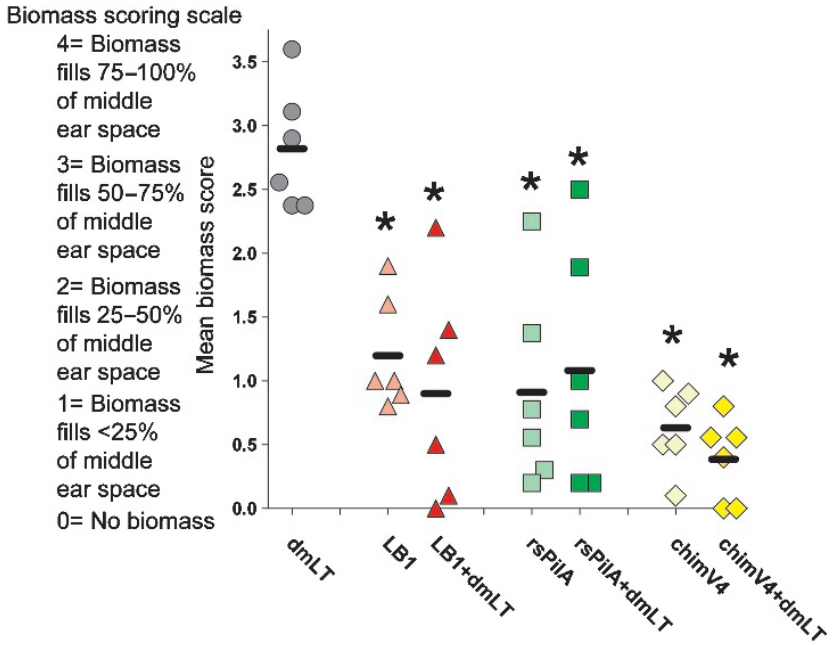

Figure 4 Mean nontypeable Haemophilus influenzae (NTHI) biomass scores for each bulla after transcutaneous immunization (TCl) following a therapeutic regimen, based on blinded evaluation and ranked on a 0 to $4+$ scale of relative residual biomass. *Statistically significant compared with receipt of $d m L T$ alone $(P<0.05)$. Receipt of LB1, rsPilA, or chimV4, admixed with $\mathrm{dmLT}$, resulted in significantly enhanced resolution of NTHI biofilms established within the middle ear space. $\mathrm{dmLT}$, double mutant of E. coli heat-labile enterotoxin; rsPilA, recombinant soluble PilA.

using this protocol within 4 days after direct challenge, $83-100 \%$ of all middle ears develop a biomass that occupies approximately $75-100 \%$ of the middle ear space. Thus, after these biomasses were established, animals were immunized by TCI to determine if the resulting antibodies could resolve these structures. At 1 week after receipt of the second dose, each middle ear was blindly ranked based on a 0 to $4+$ scale of relative biomass, wherein a score of 0 indicated that no biomass was detected within the middle ear space and $4.0+$ designated that $75-100 \%$ of the middle ear space was filled with biomass. The average biomass score for the cohort that received $\mathrm{dmLT}$ alone was 2.8 , which indicated that approximately $50-75 \%$ of the middle ear space was filled with biomass (Figure 4). In contrast, use of the therapeutic immunization regimen with LB1, rsPilA, or chimV4, alone or delivered with dmLT, resulted in a significantly reduced biomass $(P<0.05)$. The average biomass score after receipt of LB1 or rsPilA, alone or with dmLT, was between 0.9 and 1.2, and thus $75 \%$ of the biomass had resolved after TCI. Receipt of chimV4 alone or with dmLT resulted in greater reduction in the relative biomass scores to 0.6 and 0.3 , respectively. Based on relative biomass score alone, the addition of dmLT did not appear to significantly influence the outcome achieved in immunized animals. Nonetheless, overall, therapeutic immunization with the OMP P5- and Tfp-targeted immunogens elicited an immune response that resulted in a significant reduction in biomass within the middle ear.

\section{Induction of immunogen-specific $\lg G$ and $\lg A$ in serum and MEFs after immunization by a therapeutic regimen}

After TCI as a therapeutic regimen, predominantly immunogen-specific IgG was detected in serum from animals
Table 3 Geometric mean titers of immunogen-specific IgG and IgA in serum and MEF after $\mathrm{TCl}$ in a therapeutic immunization regimen

\begin{tabular}{|c|c|c|c|c|}
\hline \multirow[b]{2}{*}{ Cohort } & \multicolumn{2}{|c|}{$\begin{array}{l}\text { Immunogen-specific } \\
\text { antibody in serum }\end{array}$} & \multicolumn{2}{|c|}{$\begin{array}{l}\text { Immunogen-specific } \\
\text { antibody in MEF }\end{array}$} \\
\hline & $\lg G$ & $\operatorname{Ig} A$ & $\lg G$ & $\lg A$ \\
\hline LB1 & 40 & 40 & 20 & 10 \\
\hline $\mathrm{LB} 1+d m L T$ & $160^{*}$ & 20 & 80 & 40 \\
\hline rsPilA & 80 & 20 & 20 & 10 \\
\hline$r s P i l A+d m L T$ & $320^{*}$ & 40 & $160^{*}$ & 20 \\
\hline chimV4 & 40 & 20 & 40 & 10 \\
\hline chimV4 +dmLT & $640^{*}$ & $160^{*}$ & $320^{*}$ & 20 \\
\hline
\end{tabular}

Abbreviations: $\mathrm{dmLT}$, double mutant of $E$. coli heat-labile enterotoxin; Ig, immunoglobulin; MEF, middle ear fluid; rsPilA, recombinant soluble PilA; $\mathrm{TCl}$, transcutaneous immunization. ${ }^{*} P<0.05$ compared with respective immunogen-only titer.

that received LB1, rsPilA, or chimV4 admixed with dmLT, with GMT that was 4- to 16-times greater compared with that of animals administered respective immunogen alone (Table 3 ). Within MEFs, again, IgG was the predominant antibody isotype observed, and inclusion of dmLT in each vaccine formulation resulted in a four- to eight-fold increase in specific antibody compared with cohorts administered immunogen alone. Similar to that observed after TCI via a preventative immunization regimen (shown in Table 1), a GMT of $\geqslant 160$ was associated with enhanced resolution of biomasses within the middle ears. No consistent trend was observed with regard to biomass resolution for immunogen-specific IgA in either serum or MEFs recovered from immune animals. Thus, TCI via a therapeutic immunization regimen induced the production of both systemic and mucosal antibody (primarily IgG), which likely contributed to the observed resolution of NTHI-induced biomass from the middle ear.

\section{Resolution of MEFs after $\mathrm{TCl}$ via a therapeutic immunization regimen}

As an additional and clinically relevant assessment for the resolution of established NTHI-induced OM, each tympanic membrane was observed to document signs of OM before killing. Specifically, video otoscopy and tympanometry were performed and relative signs of inflammation and presence of MEFs behind the tympanic membrane were scored 14 days after NTHI challenge. In the cohort that received dmLT alone, 100\% (6/6) of ears were positive for the presence of MEFs (Figure 5). Receipt of LB1 alone or when delivered with dmLT induced a 17-33\% reduction in the number of ears with signs of $\mathrm{OM}$, respectively. Moreover, a 33-50\% reduction in middle ears with signs of OM was achieved after immunization with rsPilA or with chimV4 alone, respectively. It is noteworthy that immunization via a therapeutic regimen with rsPilA $+\mathrm{dmLT}$ resulted in $66 \%$ fewer ears with signs of OM, whereas no MEFs were observed behind 


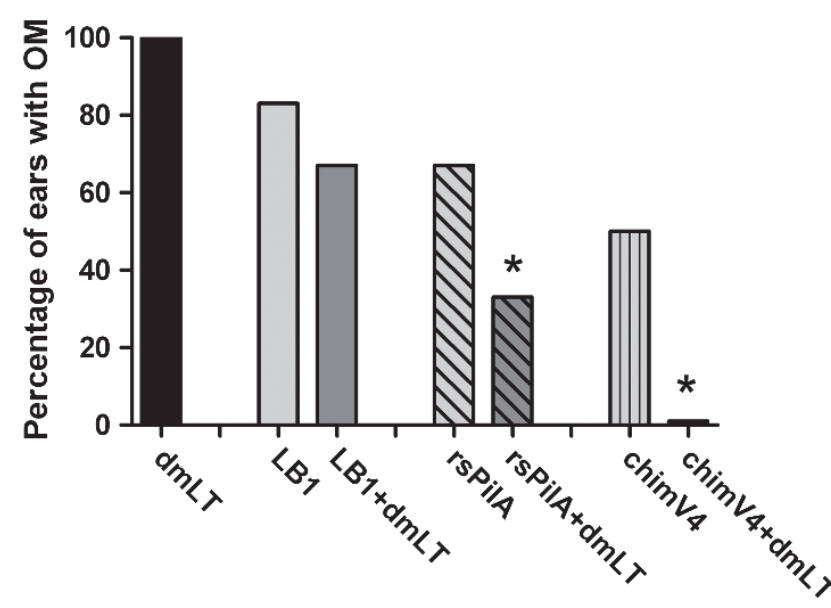

Figure 5 Percentage of middle ears with otitis media (OM) in each cohort after immunization via a therapeutic regimen, based on video otoscopy and tympanometry. *Statistically significant compared with receipt of $\mathrm{dmLT}$ alone $(P<0.05)$. Resolution of signs of OM was enhanced after receipt of LB1, rsPilA, chimV4, or LB1 +dmLT compared with receipt of $\mathrm{dmLT}$ alone. Furthermore, resolution of disease signs was significantly greater after receipt of rsPilA $+\mathrm{dmLT}$ or of chimV $4+\mathrm{dmLT}$ compared with administration of $\mathrm{dmLT}$ alone. $\mathrm{dmLT}$, double mutant of E. coli heat-labile enterotoxin; rsPilA, recombinant soluble PilA.

the tympanic membranes of animals first challenged with NTHI and then immunized with chimV4+dmLT, each a statistically significant result compared with that obtained following immunization with $\mathrm{dmLT}$ alone $(P<0.05)$. Unlike our observations for biomass reduction alone, wherein we were unable to detect the influence of dmLT, here our data showed a greater reduction in signs of OM after TCI with any of the three immunogens admixed with dmLT, compared with immunogen alone. Therefore, TCI after direct challenge of the middle ear resulted in resolution of signs of ongoing NTHI-induced OM in addition to mediating a marked reduction in resident biomass within the middle ear.

\section{Migration of DCs from the pinnae}

Last, we examined the migration of DCs from the pinna after TCI to identify potential sites of immune induction after immunization by this route. To do so, the amine-reactive dye carboxyfluoroscein succinimidyl ester (CFSE) was applied to the pinnae along with each vaccine formulation to allow for discrimination among cells resident within lymphoid tissues and DCs that had migrated to these sites from the pinnae. ${ }^{25,26}$ We observed that within $1 \mathrm{~h}$ after TCI, only $13.6 \%$ of cells isolated from the nasal-associated lymphoid tissue (NALT) of animals immunized with adjuvant alone were $\mathrm{CFSE}^{+} \mathrm{CD} 11 \mathrm{c}^{+}$ (Figure 6a). In contrast, TCI with either rsPilA, LB1, or chimV4 each delivered with dmLT resulted in a 2.5- to 5.4-fold relative increase (37.2, 34.4, and $72.9 \%$, respectively) in the percentage of $\mathrm{CFSE}^{+}$DCs detected within this lymphoid aggregate (Figure 6b-d). $\mathrm{CFSE}^{+} \mathrm{CD} 11 \mathrm{c}^{+}$DCs were also detected in the axillary and brachial lymph nodes, although only 1.1- to 2.0fold more $\mathrm{CFSE}^{+}$DCs were observed in lymph nodes after TCI with any immunogen vs. the adjuvant alone (data not shown).
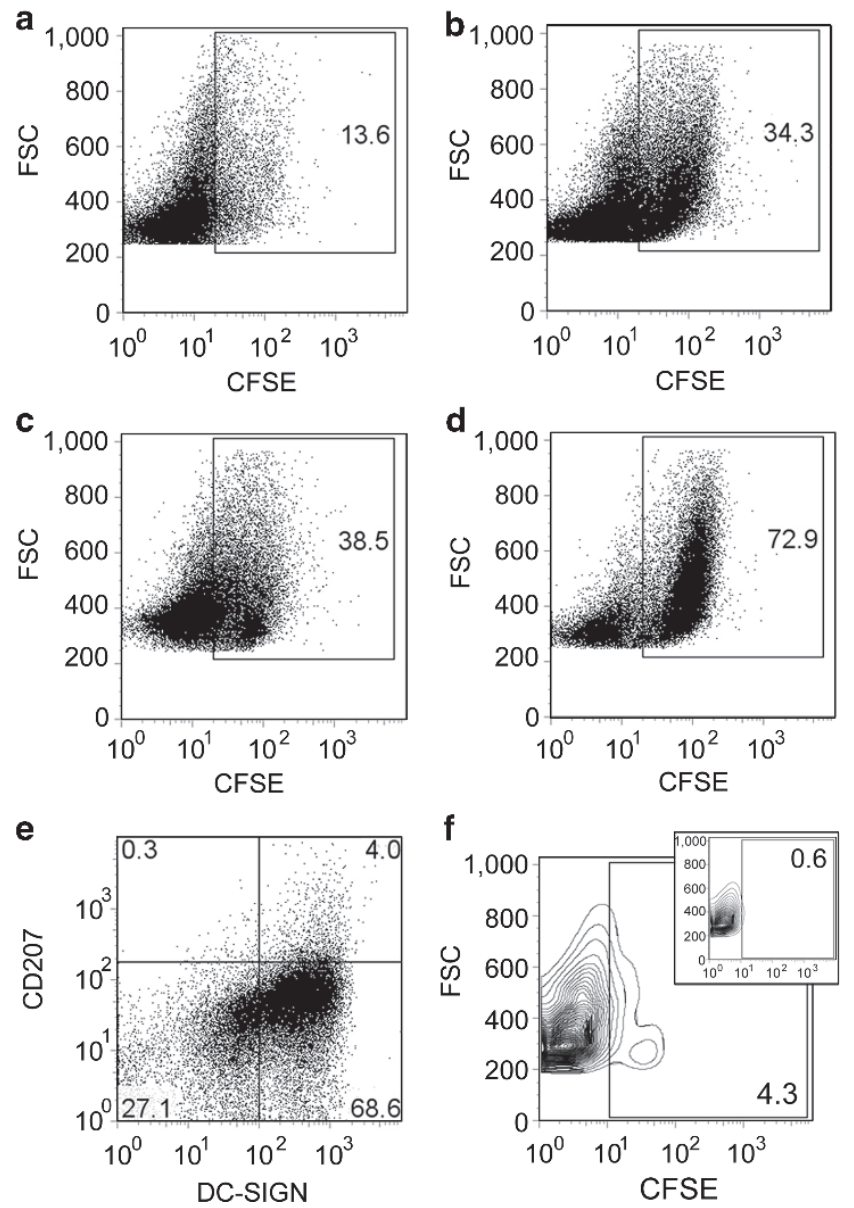

Figure 6 Migration of dendritic cells (DCs) from the pinnae to the nontypeable Haemophilus influenzae (NALT) after transcutaneous immunization (TCl) and discrimination of cutaneous DC cell type.

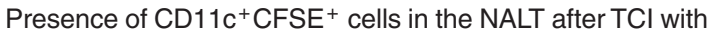
(a) dmLT alone, (b) rsPilA $+d m L T$, (c) $L B 1+d m L T$, or (d) chimV4 $+d m L T$ was detected by flow cytometry. Numbers in each box represent the percentage of $\mathrm{CFSE}^{+}$cells within each sample. (e) $\mathrm{CFSE}^{+}$cells were identified to be DC-SIGN ${ }^{+}$dermal DCs. (f) Bone marrow-derived DCs activated with chimV4+dmLT ex vivo and then injected subdermally into pinnae migrated to the NALT, whereas dmLT-activated DCs did not (inset). CFSE, carboxyfluoroscein succinimidyl ester; dmLT, double mutant of $E$. coli heat-labile enterotoxin; FSC, forward scatter; rsPilA, recombinant soluble PilA.

No difference in the percentage of $\mathrm{CFSE}^{+} \mathrm{CD} 11 \mathrm{c}^{+} \mathrm{DCs}$ within the cervical or mediastinal lymph nodes or spleen of animals that received an immunogen and those immunized with dmLT alone was found at this time point. These data suggested that DCs within the pinnae sampled the NTHI adhesin-derived immunogens that had been applied to the surface of the pinnae and were induced to mature. DC maturation resulted in the preferential migration of these cells to primarily the NALT, and also the axillary and brachial lymph nodes.

Further analysis was performed to distinguish between the DC types that migrated to the lymphoid tissues. We specifically focused on discrimination between Langerhan's cells vs. dermal DCs, the primary antigen-presenting cells found within the skin. A total of $\sim 69 \%$ of $\mathrm{CFSE}^{+}$cells within the NALT were positively 
labeled with antibody directed against dendritic cell-specific intercellular adehsion molecule-3 grabbing integrin (DC-SIGN), specific for dermal DCs, ${ }^{27}$ as opposed to $0.3 \%$ of cells that labeled positively for CD207, which is selectively expressed by Langerhan's cells ${ }^{28}$ (Figure 6e). Therefore, these data indicated that the primary antigen-presenting cells involved in induction of the immune responses observed herein were DCs.

To further confirm that the positive signal described above was because of the migration of DCs to lymphoid tissues and not because of diffusion of the dye alone through the lymphatics to that site, we labeled bone marrow-derived DCs with CFSE, activated them with chimV $4+\mathrm{dmLT}$ or $\mathrm{dmLT}$ alone in vitro, and then injected the DCs intradermally into the pinnae. After $1 \mathrm{~h}$, a population of $\mathrm{CFSE}^{+}$cells was detected within the NALT after receipt of DCs activated with chimV4 + dmLT (Figure 6f), but not when activated with dmLT alone (Figure 6f, inset). Moreover, $4.3 \%$ of the cells isolated from the NALT were $\mathrm{CFSE}^{+}$, whereas $\leqslant 0.6 \%$ was observed within the cervical, axillary, and brachial lymph nodes and spleen (data not shown). Thus, the migration of DCs resident within the pinnae after TCI and those instilled into the pinnae after in vitro activation were similar, a result that suggested preferential homing to this local lymphoid aggregate as mediated via local cytokine/chemokine signals. Collectively, our tracking data demonstrated that immunogen-activated DCs within the pinnae migrated primarily to a proximal lymphoid aggregate, the NALT. Moreover, the cell type activated after TCI was the dermal DC, which likely had a critical role in the initiation of the protective immune response observed herein.

\section{DISCUSSION}

$\mathrm{OM}$ is a prevalent disease of children worldwide, and although commonly managed by prescription of antibiotics and surgical intervention where available, the incidence and cost attributed to this disease is substantial. ${ }^{2}$ Moreover, the emergence of multiple antibiotic-resistant bacteria, including strains of NTHI, is a cause for concern. ${ }^{29}$ Thus, immunization against OM has the potential to alleviate this socioeconomic burden by prevention or resolution of disease. At present, a licensed 10-valent pneumococcal capsular conjugate vaccine wherein NTHI protein D serves as a carrier molecule (Synflorix, GlaxoSmithKline, Rixensart, Belgium) has demonstrated $35.3 \%$ protective efficacy against NTHI-induced OM after parenteral immunization of children in a clinical trial in Slovakia and the Czech Republic. ${ }^{30}$ Although shown as a secondary outcome measure, this trial nonetheless demonstrated, for the first time, that parenteral delivery of a NTHI-derived antigen could provide protection against OM due to NTHI. To increase the modest coverage observed against NTHI-induced OM, examination of additional NTHI-specific targets is needed.

We have focused our vaccine development efforts on two of multiple adhesins expressed by NTHI, OMP P5 and Tfp, and recently utilized an established chinchilla model of viral-bacterial superinfection shown to be predictive of the aforementioned clinical trial outcome. ${ }^{7,31}$ Collectively, these preclinical data demonstrated that antibody induced by parenteral immunization with NTHI OMP P5- and Tfp-derived vaccine candidates (called LB1, rsPilA, and chimV4) provides significant protection against experimental NTHI-induced OM. ${ }^{6}$ We now sought to expand our vaccine delivery routes to include noninvasive administration regimens. Currently, children in the United States receive up to 25 vaccines by injection during the first 2 years of life. ${ }^{32}$ This can be cause for concern by parents who may ultimately delay or refuse immunization of their child. Also, although not of considerable concern in developed countries, the reuse of needles in developing countries poses serious risk for transmission of blood-borne diseases. ${ }^{33,34}$ Thus, although vaccination by injection is proven to be extremely effective as a preventative intervention, development of alternative delivery strategies for current or future formulations could potentially serve as equally effective means to induce protective immunity while simultaneously addressing bottlenecks associated with injectable vaccines.

TCI is a simple and noninvasive method for vaccine administration. In addition to the ease of delivery, both systemic and mucosal immunity is induced by this regimen. OM is a disease of the uppermost respiratory tract mucosa, and thus the potential to initiate mucosal immunity, particularly in the nasopharynx where NTHI normally reside, holds promise to limit or prevent its onset. We focused on the chinchilla pinnae as an easily accessible site for administration that did not require manipulation, such as shaving, before delivery. Moreover, as there is greater appreciation for compartmentalization within the mucosal immune system because of homing of immune cells that results in the most powerful response induced at the site proximal to delivery, ${ }^{35,36}$ we wanted to favor development of an immune response in proximity to the NP and middle ear. This premise is supported by work in a murine host, where after TCI, the greatest cytotoxic lymphocyte activity was detected by cells isolated from lymphoid tissues in close proximity to site of administration whereas less activity was observed by cells from distal tissues. ${ }^{11}$

A proof-of-concept study demonstrated that TCI induced an immunogen-specific immune response in the chinchilla host, and that bilateral (both pinnae) administration induced more robust immunity compared with unilateral delivery (one pinna; data not shown). Furthermore, upon challenge with NTHI, we observed clearance of NTHI from the NP and middle ear. Herein, we expanded upon that preliminary study to examine the protection afforded by TCI with the OMP P5-derived candidate LB1, the Tfp-directed candidate rsPilA, and the chimeric OMP P5- plus Tfp-directed immunogen chimV4 when delivered with dmLT, a potent adjuvant.

Our current data demonstrated that TCI before NTHI challenge with any of the three immunogens induced the production of both immunogen-specific IgA and IgG and although clearance of NTHI was associated with the production of both antibody isotypes, a greater relative quantity of IgG was consistently observed. NTHI possess the iga gene that encodes an IgA1 protease, and up to a third of strains have an additional gene (igaB) that encodes a second IgA protease. ${ }^{37,38}$ Whereas $\operatorname{IgA}$ is the conventional antibody isotype detected at mucosal 
surfaces, mucosal IgG, either produced locally or present via transudation from serum, is also known to contribute to protection against mucosal infection. ${ }^{35}$ We observed a correlation between antibody GMT of $\geqslant 160$ and enhanced resolution of disease after TCI via use of a preventative or therapeutic regimen. Protection against NTHI-induced OM is primarily antibody mediated, although the contribution of T-cell-mediated mechanism cannot be ruled out, as it is required for clearance of NTHI from the rat lung. ${ }^{39}$ The data presented herein demonstrated that TCI induced the production of antibodies that targeted adhesins expressed by NTHI and, as such, was likely the primary mechanism for eradication of the bacterium from the nasopharynx and middle ear.

TCI before bacterial challenge with rsPilA, LB1, or chimV4 delivered with $\mathrm{dmLT}$ resulted in a rapid reduction in the bacterial load of NTHI within the NP of immune chinchillas, in addition to a reduction in the percentage of culture-positive animals per cohort, compared with receipt of immunogen or adjuvant alone. Within middle ears, whereas an increase in bacterial concentration is typically observed after direct challenge because of initial bacterial replication, ${ }^{24}$ only a modest initial increase in CFU NTHI was observed in cohorts that received any of the three immunogens plus dmLT and was followed by a rapid elimination of bacteria from the middle ear. These data clearly demonstrated that TCI with OMP P5 or Tfp-targeted immunogens with dmLT induced an efficacious immune response. Among these three cohorts, differences were observed specific to the time to clear NTHI from the NP and middle ear after challenge. It is known that OMP P5 is constitutively expressed and is important for enabling NTHI to establish colonization in the NP. ${ }^{40}$ NTHI Tfp are temporally expressed and necessary for NTHI to maintain long-term colonization at this site. ${ }^{22}$ Moreover, these adhesins are utilized for both adherence and biofilm formation within the middle ear. Thus, the presence of antibody specific to each adhesin, or both adhesins, likely served to prevent NTHI adherence, which facilitated elimination of NTHI from the NP and middle ear upon challenge.

$\mathrm{OM}$ can be a chronic and/or recurrent disease, a consequence of biofilms established by NTHI within the middle ear. Based on the efficacy afforded by immunization with the OMP P5- and Tfp-targeted immunogens before bacterial challenge, we wondered whether TCI after NTHI challenge might also effectively resolve OM by eradicating an already established NTHI biofilm from the middle ear. Immunization via a therapeutic regimen resulted in a reduction in the signs of OM based on a clinically relevant scoring system, in addition to overall resolution of an established NTHI biomass in the middle ear as determined by blinded gross examination of the middle ear space. Whereas a significant reduction in biomass was grossly observed for all immune animals compared with those that received dmLT alone, only cohorts that received rsPilA or chimV4 admixed with dmLT demonstrated a significant reduction in signs of disease as determined by video otoscopy and tympanometry. The two techniques, one of gross examination and the other of observation for signs of disease, do not discredit each other. Rather, they indicated that whereas TCI via a therapeutic route resulted in reduction of middle ear biomass, MEFs had yet to drain through the Eustachian tube or be absorbed by the middle ear mucosa in all animals, although overall signs of OM were reduced. Therapeutic vaccines for control of other viral or bacterial infections have been examined in experimental and clinical trials with promising results, ${ }^{41-43}$ and currently in use is a therapeutic canine melanoma vaccine to combat oral melanoma. ${ }^{44}$ Thus, the strategy to resolve active disease by therapeutic vaccination is promising.

An additional observation from each study presented herein was the enhanced immune response and resultant bacterial clearance by formulation of each immunogen with dmLT, compared with administration of immunogen alone. Derivatives of cholera toxin and E. coli heat-labile enterotoxin have shown utility as adjuvants for mucosal and cutaneous immunization regimens, wherein the toxicity associated with mucosal application of each holotoxin is not observed. ${ }^{45}$ Specific to dmLT, the engineered amino-acid substitutions at position 192 inactivates a trypsin cleavage site and at position 211 modifies a potential pepsin cleavage site. ${ }^{16}$ The resultant molecule is insensitive to proteolysis by either enzyme, as by design. Furthermore, no toxicity is observed in a patent mouse enterotoxicity model. ${ }^{16}$ Although the exact mechanism is undefined, it is believed that adjuvant activity is an overall outcome of the activation of various cell types, including DCs. ${ }^{46,47}$

We also examined the role of cutaneous DCs in the observed rapid and protective immune response. Our data demonstrated that TCI with the OMP P5- and Tfp-targeted immunogens delivered with dmLT induced the maturation and subsequent migration of DCs resident within the pinnae, a response not observed after application of dmLT alone. As a complimentary approach, DCs activated ex vivo and then injected subdermally into the pinnae exhibited similar maturation and migration characteristics as in vivo-activated DCs. By each approach, immunogen-activated DCs migrated primarily to the NALT, an immune inductive site, ${ }^{48}$ likely because of local cytokine/ chemokine signaling. Migratory cells within the NALT were identified to be predominantly dermal DCs, not Langerhan's cells, based on the expression of DC-SIGN vs. CD207. Although not completely defined, it is proposed that Langerhan's cells are responsible for tolerogenic immunity, i.e., to self-antigens, whereas dermal DCs are involved in development of protective immunity ${ }^{49}$ Moreover, compared with Langerhan's cells, dermal DCs exhibit faster migration and populate lymph nodes in areas closer to B-cell follicles, ${ }^{50}$ facilitating antigen presentation to $\mathrm{B}$ cells and initiation of immune responses. Therefore, TCI served to engage dermal DCs within the chinchilla pinnae that facilitated the development of a local and systemic immune response. We continue to examine the contribution of additional innate immune effector to the rapid resolution observed herein.

As we continue to develop TCI as a delivery method, it is important to consider the practical application of this method to humans, particularly to very young children. To promote development of a mucosal immune response in the uppermost airway of a child, it is rational to propose vaccine application to skin behind the ear or back of the neck. There is evidence that 
the Waldeyer's tonsillar ring in the neck and pharynx functions similar to the rodent NALT, and thus the potential exists to induce an immune response within lymphoid tissues proximal to the human nasopharynx and middle ear, as desired. Furthermore, to facilitate widespread application, the TCI method must be simple and inexpensive, preferably deviceless, and yet be able to efficiently deliver the formulation to the dermal DCs through the stratum corneum. Whereas there are multiple strategies in development to breach this protective barrier, ${ }^{51}$ we suggest that direct application of a gel or cream on to the skin also has the potential to hydrate the stratum corneum, restrict diffusion of the antigen, and promote long-term sampling by DCs.

In summary, TCI served to engage dermal DCs within the chinchilla pinnae that resulted in the development of a local mucosal, in addition to systemic, immune response. Administration of the NTHI OMP P5- and Tfp-directed immunogens admixed with dmLT was efficacious to both prevent experimental NTHI-induced OM and to resolve active OM. These data therefore demonstrated that TCI is an effective way to immunize against experimental NTHI-induced OM and has potential application to prevent other diseases of the respiratory tract due to NTHI. Moreover, the reduced costs associated with noninvasive routes of immunization such as TCI hold great promise in terms of expanding the use of vaccines to prevent OM beyond the boundaries of developed countries.

\section{METHODS}

\section{Animals}

To examine the efficacy of TCI utilized as a preventative immunization strategy, 70 adult chinchillas (Chinchilla lanigera; Rauscher's Chinchilla Ranch, LaRue, $\mathrm{OH}$; mean mass $662 \pm 15 \mathrm{~g}$ ) with no evidence of middle ear disease as determined by video otoscopy (MedRx, Largo, FL) and tympanometry (EarScan, Murphy, NC) were enrolled and divided into 7 cohorts of 10 animals each. To test TCI as a therapeutic immunization strategy, 21 adult chinchillas $(625 \pm 23 \mathrm{~g})$ were enrolled and divided into 7 cohorts of 3 animals each. Animal care and all procedures were performed in concordance with institutional and federal guidelines, and were conducted under an approved protocol.

\section{Immunogens and adjuvant}

NTHI OMP P5 was observed expressed by $100 \%$ of a panel of middle ear and nasopharyngeal NTHI isolates collected from children with chronic OM.52 Multiple algorithmic analyses of the deduced amino-acid sequence of OMP P5 predict four surface-exposed regions within the $\mathrm{N}$-terminus of this adhesin, ${ }^{53,54}$ and within the third surface-exposed region is a 19 -mer B-cell epitope that is incorporated into LB1. ${ }^{55}$ Among NTHI strains tested, there is limited amino-acid sequence diversity within this moiety that allows for segregation of isolates into three groups, $76 \%$ of which cluster into one majority group. The immunogen LB1 is a 40-mer synthetic chimeric peptide comprising the aforementioned 19-mer B-cell epitope from OMP P5 that is colinearly synthesized with a T-cell promiscuous epitope from measles virus fusion protein, incorporated to be broadly permissive in binding to major histocompatibility complex class II molecules.

PilA, the majority subunit of NTHI Tfp, has minimal deduced aminoacid sequence diversity. Among a panel of 23 isolates tested, the product of the pilA gene exhibits $\geqslant 81 \%$ sequence identity to that of NTHI strain $86-028 N{ }^{56}{ }^{56}$ Recombinant, soluble PilA (rsPilA) is a 122 aminoacid recombinant protein that represents a mature, N-terminally truncated PilA subunit of the NTHI Tfp. ${ }^{6,22,56}$ ChimV4 is a novel, chimeric immunogen wherein modified rsPilA serves as an immunogenic carrier for a slightly larger (24 amino acids) variant of the OMP P5 B-cell epitope described within the immunogen LB1. ${ }^{6}$ A double mutant form of E. coli heat-labile enterotoxin, called LT(R192G-L211A) and abbreviated $\mathrm{dmLT}$, wherein glycine is substituted for arginine at position 192 and alanine is substituted for lysine at position 211 , served as the adjuvant. ${ }^{16}$ The amino-acid substitutions render dmLT nontoxic while maintaining its adjuvant properties.

\section{NTHI strain}

NTHI strain 86-028NP was isolated from the nasopharynx of a child undergoing tympanostomy and tube insertion for chronic $\mathrm{OM}$ at Nationwide Children's Hospital, Columbus, $\mathrm{OH}$. This strain has been characterized and extensively used in chinchilla models of OM and a rat model of pulmonary clearance. ${ }^{7,24,31,57}$

\section{Immunization via a preventative regimen and NTHI challenge}

TCI via a preventative immunization regimen was performed as follows: both pinnae of each alert animal were hydrated for $5 \mathrm{~min}$ by placement of gauze soaked in sterile, pyrogen-free $0.9 \%$ sodium chloride (Hospira, Lake Forest, IL) on the inner surface before vaccination. The inner surface of each pinna was then blotted with dry gauze and $50 \mu \mathrm{l}$ of each vaccine formulation was applied using a pipet. The pinnae were then folded in half and opposing surfaces gently rubbed together. Formulations consisted of $10 \mu \mathrm{g}$ LB1, rsPilA, or chimV4 delivered alone or admixed with $10 \mu \mathrm{g}$ LT(R192G-L211A), also called "dmLT," or $10 \mu \mathrm{g}$ dmLT alone. Two doses were delivered at weekly intervals. NTHI challenge was performed as previously described. ${ }^{24}$ Briefly, at 1 week after receipt of the second immunizing dose, all chinchillas were challenged intranasally with $10^{8} \mathrm{CFU}$ NTHI strain $86-028 \mathrm{NP}$ delivered in $0.2 \mathrm{ml}$ pyrogen-free saline divided equally between the nares, as well as transbullarly with 1,000 CFU NTHI delivered in $0.3 \mathrm{ml}$ sterile pyrogen-free saline per bulla. Challenge doses were confirmed by plate count.

\section{NTHI challenge and immunization via a therapeutic strategy}

TCI following a therapeutic regimen involved challenge of all chinchillas exclusively transbullarly with 1,000 CFU NTHI strain 86-028NP before immunization. At 4 days after NTHI challenge, animals were immunized by TCI as described above. Formulations consisted of $10 \mu \mathrm{g} \mathrm{LB1}$, rsPilA, or chimV4 delivered alone or admixed with $10 \mu \mathrm{g}$ dmLT or $10 \mu \mathrm{g} \mathrm{dmLT}$ alone and were delivered twice at weekly intervals. At 1 week after receipt of the second immunizing dose, all animals were killed.

\section{Histology of chinchilla pinnae}

Pinnae were collected and trimmed to the central $2.5 \mathrm{~mm}^{2}$ portion. The pinna was then cut into four strips of equal size and fixed in $2 \% \mathrm{w} / \mathrm{v}$ paraformaldehyde in $0.1 \mathrm{~m}$ phosphate buffer, $\mathrm{pH} 7.4$, for $24 \mathrm{~h}$. Strips were processed for routine histology and embedded in paraffin. Serial sections $(5 \mu \mathrm{m})$ were cut and tissue stained with hematoxylin and eosin.

\section{Collection of blood and mucosal secretions}

Blood for serum was collected via cardiac puncture before immunization (pre) and 1 week after receipt of the second immunizing dose (immune) for all animals immunized via a preventative regimen. Animals immunized via a therapeutic regimen were bled before NTHI challenge and at study end.

NP lavages were performed on animals immunized via a preventative regimen before immunization, 1 week after receipt of the final dose, and on days $3,7,10$, and 14 after bacterial challenge by passive inhalation of $500 \mu \mathrm{l}$ sterile pyrogen-free saline as previously described. ${ }^{24}$

Video otoscopy (using a $0^{\circ}, 3$-inch probe connected to a digital camera system) to monitor signs of tympanic membrane inflammation and/or presence of fluid within the middle ear space was performed and overall signs of OM were rated on a scale of 0 to $4+$ as previously described.7,24,31 Middle ears with a score of $\geqslant 2.0$ were always considered positive for $\mathrm{OM}$ as $\mathrm{MEF}$ is visible behind the tympanic membrane. Each middle ear was 
considered independent, and for each cohort, the percentage of middle ears with OM was calculated.

Epitympanic taps to retrieve middle ear effusions were performed on any chinchilla whose tympanic membrane was rated as $\geqslant 2.5$ on a scale of 0 to +4.0 . Epitympanic taps were not performed on ears ranked 2.0, due potential for perforation of the tympanic membrane to retrieve the low volume of MEF. NP lavage and MEFs were serially diluted and plated onto chocolate agar supplemented with $15 \mu \mathrm{g}$ ampicillin per ml medium or chocolate agar, respectively, to semiquantitate CFU NTHI per ml fluid type. The mean CFU NTHI per ml fluid was reported for each cohort.

\section{Evaluation of middle ear biomass}

Upon killing of animals immunized after NTHI challenge, the inferior bullae from each animal were dissected, opened to reveal the middle ear space, and washed with $1 \mathrm{ml}$ sterile, pyrogen-free saline to remove residual MEF and loosely adherent biomass. The bullae and remaining adherent biomass were then imaged with a digital camera. The images from the left and right bulla were scrambled and scored by nine blinded observers who ranked the relative residual biomass on a 0 to $4+$ scale, wherein $0=$ no biomass; $1=$ biomass fills $<25 \%$ of middle ear space; $2=$ biomass fills $25-50 \%$ of middle ear space; $3=$ biomass fills $50-75 \%$ of middle ear space; and $4=$ biomass fill $75-100 \%$ of middle ear space. The mean biomass score for each bulla was reported.

\section{Enzyme-linked immunosorbent assay}

Enzyme-linked immunosorbent assay was performed on serum to detect immunogen-specific IgG and IgA in immune serum and MEFs. Samples were incubated in LB1-, rsPilA-, or chimV4-coated wells $(0.2 \mu$ g protein per well) for $3 \mathrm{~h}$ at $25^{\circ} \mathrm{C}$ and antibody was detected with horseradish peroxidase-conjugated goat anti-rat IgG or IgA (Bethyl Laboratories, Montgomery, TX). Color was developed with 3,3',5,5' -tetramethylbenzidine (Pierce Biotechnology, Rockford, IL). Reciprocal titers were defined as the dilution that yielded an $\mathrm{OD}_{450 \mathrm{~nm}}$ value of 0.1 above control wells that were incubated without sample fluids. Assays were performed a minimum of three times and reciprocal titers reported as the GMT.

\section{Flow cytometry to detect recognition of native proteins on NTHI}

To detect the recognition of native OMP P5 and Tfp as expressed on the surface of NTHI by antibodies in NP lavage fluids, flow cytometric analysis was performed. NTHI strain 86-028NP was cultured overnight on chocolate agar, and then suspended in brain heart infusion broth supplemented with $2 \mu \mathrm{g}$ each of $\beta$-NAD and heme (Sigma Aldrich, St Louis, MO). The optical density was adjusted to 0.6 at $490 \mathrm{~nm}$, further diluted 1:6 in fresh supplemented brain heart infusion broth, and the culture incubated static for $3 \mathrm{~h}$ at $37^{\circ} \mathrm{C}$, previously shown to be time of maximal pil promoter activity. ${ }^{56} \mathrm{NTHI}$ were then incubated with a $1: 10$ dilution of pooled clarified NP lavage fluids for $1 \mathrm{~h}$ at $37^{\circ} \mathrm{C}$ on a rotational rocker. Chinchilla immunoglobulins were detected with Protein A conjugated to fluorescein isothiocyanate (Zymed, South San Francisco, CA). A total of 20,000 events were collected per sample using a FACSCalibur flow cytometer (Becton Dickinson, Franklin Lakes, NJ) and data analyzed with FlowJo software (Tree Star, Ashland, OR). Assays were performed a minimum of three times.

\section{In vivo migration of DCs from the pinnae}

To track the migration of chinchilla DCs from the pinnae to lymphoid tissues, we applied the amine-reactive vital dye, CFSE (Invitrogen, Carlsbad, CA) with $\mathrm{LB} 1+\mathrm{dmLT}$, rsPilA + dmLT, chimV4+dmLT, or dmLT alone to passively label cells that sampled the vaccine formulations after delivery. ${ }^{25,26}$ To confirm a minimal contribution of nonspecific activation of DCs by the dye itself, CFSE alone was also applied. At $1 \mathrm{~h}$ after TCI with vaccine formulations and CFSE, animals were killed and the cervical, brachial, axillary, and mediastinal lymph nodes were removed in addition to the spleen. The NALT was also removed. Tissues were crushed into Dulbecco's phosphate-buffered saline without calcium or magnesium (Mediatech, Manassas, VA) and cells passed through a $40 \mu \mathrm{m}$ cell strainer.
The spleen was also crushed, strained, and the cells centrifuged with Ficoll-Paque. DCs were labeled with mouse anti-rat CD11c-Alexa fluor 647 (AbD Serotec, Raleigh, NC) and CD11c ${ }^{+} \mathrm{CFSE}^{+}$cells were detected by flow cytometry. A total of 10,000 events were collected.

To discriminate between Langerhan's cells and dermal DCs, $10^{6}$ cells from each lymphoid tissue cell suspension were incubated with mouse anti-human DC-SIGN-APC, and then permeabilized and incubated with mouse anti-human CD207-PE (R\&D Systems, Minneapolis, MN) or appropriate isotype control antibody and assayed via flow cytometry. A total of 10,000 events were collected. Assays were performed a minimum of three times and representative scatter plot shown.

To confirm that the $\mathrm{CFSE}^{+}$signal detected within the NALT was specifically because of the migration of DCs from the pinnae and not because of diffusion of the dye within the lymphatics, we investigated whether in vitro-activated, bone marrow-derived DCs would similarly follow homing signals to the NALT when applied to the pinnae. Thus, chinchilla bone marrow cells were harvested and cultured in the presence of $40 \mathrm{ng}$ each of human recombinant granulocyte-macrophage colony-stimulating factor and interleukin-4 (R\&D Systems) per ml to induce differentiation of precursor cells as previously described. ${ }^{26}$ After 6 days, the immature DCs were labeled with $10 \mu \mathrm{M}$ CFSE before incubation for $30 \mathrm{~min}$ with either $10 \mu \mathrm{g}$ chimV4 plus $10 \mu \mathrm{g} \mathrm{dmLT}$ or $10 \mu \mathrm{g}$ dmLT alone. The treated DCs were washed to remove unbound immunogen, adjusted to $10^{6}$ cells per $100 \mu \mathrm{l}$, and injected intradermally to alert chinchillas in a volume of $50 \mu \mathrm{l}$ per pinna. The animals were killed $1 \mathrm{~h}$ later, and the lymphoid tissues were processed as described above. $\mathrm{CFSE}^{+}$cells were detected by flow cytometry. A total of 10,000 viable events were collected for each of three independent assays and representative plots are shown.

\section{Statistics}

Statistical differences between antibody titers, bacterial counts in NP lavage fluids, and MEFs were determined using Kruskal-Wallis oneway analysis of variance on ranks and Dunn's method for multiple comparisons. A $P$-value of $\leqslant 0.05$ was considered significant. Significant differences in relative biomass among cohorts and percentage of middle ears with $\mathrm{OM}$ were assessed by a repeated measures analysis of variance and Bonferroni's multiple comparison test. A $P$-value of $\leqslant 0.05$ was considered significant.

\section{ACKNOWLEDGMENTS}

This work was supported by R01 DC003915 and DC007464 to L.O.B. We thank Glen McGillivary, Amanda Dickson, Joseph Jurcisek, and Dana Staffen for technical assistance, Dr Santiago Partida-Sanchez for dendritic cell experimental design, and Jennifer Neelans for article preparation.

\section{DISCLOSURE}

The authors declared no conflict of interest.

(C) 2011 Society for Mucosal Immunology

\section{REFERENCES}

1. Foxwell, A.R., Kyd, J.M. \& Cripps, A.W. Nontypeable Haemophilus influenzae: pathogenesis and prevention. Microbiol. Mol. Biol. Rev. 62, 294-308 (1998).

2. Kaplan, B., Wandstrat, T.L. \& Cunningham, J.R. Overall cost in the treatment of otitis media. Pediatr. Infect. Dis. J. 16 (2 Suppl), S9-S11 (1997).

3. Baldwin, R.L. Effects of otitis media on child development. Am. J. Otol. 14, 601-604 (1993).

4. Murphy, T.F. Current and future prospects for a vaccine for nontypeable Haemophilus influenzae. Curr. Infect. Dis. Rep. 11, 177-182 (2009).

5. Bakaletz, L.O. Peptide and recombinant antigens for protection against bacterial middle ear infection. Vaccine 19, 2323-2328 (2001).

6. Novotny, L.A. et al. Epitope mapping immunodominant regions of the PilA protein of nontypeable Haemophilus influenzae (NTHI) to facilitate the design of two novel chimeric vaccine candidates. Vaccine $\mathbf{2 8 , 2 7 9 - 2 8 9}$ (2009). 
7. Kennedy, B.J., Novotny, L.A., Jurcisek, J.A., Lobet, Y. \& Bakaletz, L.O. Passive transfer of antiserum specific for immunogens derived from a nontypeable Haemophilus influenzae adhesin and lipoprotein D prevents otitis media after heterologous challenge. Infect. Immun. 68, 2756-2765 (2000).

8. Warger, T., Schild, H. \& Rechtsteiner, G. Initiation of adaptive immune responses by transcutaneous immunization. Immunol. Lett. 109, 13-20 (2007).

9. Giudice, E.L. \& Campbell, J.D. Needle-free vaccine delivery. Adv. Drug. Deliv. Rev. 58, 68-89 (2006).

10. Maeba, S. et al. Transcutaneous immunization with a 40-kDa outer membrane protein of Porphyromonas gingivalis induces specific antibodies which inhibit coaggregation by $P$. gingivalis. Vaccine $\mathbf{2 3}$, 2513-2521 (2005)

11. Belyakov, I.M., Hammond, S.A., Ahlers, J.D., Glenn, G.M. \& Berzofsky, J.A. Transcutaneous immunization induces mucosal CTLs and protective immunity by migration of primed skin dendritic cells. J. Clin. Invest. 113, 998-1007 (2004).

12. Vogt, A. et al. Transcutaneous anti-influenza vaccination promotes both CD4 and CD8 T cell immune responses in humans. J. Immunol. 180, 1482-1489 (2008).

13. Rollenhagen, J.E. et al. Transcutaneous immunization with toxincoregulated pilin $\mathrm{A}$ induces protective immunity against Vibrio cholerae $\mathrm{O}$ El Tor challenge in mice. Infect. Immun. 74, 5834-5839 (2006).

14. Ghose, C. et al. Transcutaneous immunization with Clostridium difficile toxoid $\mathrm{A}$ induces systemic and mucosal immune responses and toxin A-neutralizing antibodies in mice. Infect. Immun. 75, 2826-2832 (2007).

15. Skountzou, I., Quan, F.S., Jacob, J., Compans, R.W. \& Kang, S.M. Transcutaneous immunization with inactivated influenza virus induces

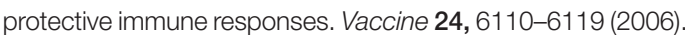

16. Summerton, N.A. et al. Toward the development of a stable, freeze-dried formulation of Helicobacter pylori killed whole cell vaccine adjuvanted with a novel mutant of Escherichia coli heat-labile toxin. Vaccine 28, 1404-1411 (2010).

17. Mishra, D., Mishra, P.K., Dubey, V., Nahar, M., Dabadghao, S. \& Jain, N.K. Systemic and mucosal immune response induced by transcutaneous immunization using Hepatitis B surface antigen-loaded modified liposomes. Eur. J. Pharm. Sci. 33, 424-433 (2008).

18. Murphy, T.F., Bakaletz, L.O. \& Smeesters, P.R. Microbial interactions in the respiratory tract. Pediatr. Infect. Dis. J. 28 (10 Suppl), S121-S126 (2009).

19. Hall-Stoodley, L. et al. Direct detection of bacterial biofilms on the middleear mucosa of children with chronic otitis media. JAMA 296, 202-211 (2006).

20. Ehrlich, G.D. et al. Mucosal biofilm formation on middle-ear mucosa in the chinchilla model of otitis media. JAMA 287, 1710-1715 (2002).

21. Gallaher, T.K., Wu, S., Webster, P. \& Aguilera, R. Identification of biofilm proteins in non-typeable Haemophilus influenzae. BMC Microbiol. 6, 65 (2006).

22. Jurcisek, J.A. \& Bakaletz, L.O. Biofilms formed by nontypeable Haemophilus influenzae in vivo contain both double-stranded DNA and type IV pilin protein. J. Bacteriol. 189, 3868-3875 (2007).

23. Tan, G. et al. Hydration effects on skin microstructure as probed by highresolution cryo-scanning electron microscopy and mechanistic implications to enhanced transcutaneous delivery of biomacromolecules. J. Pharm. Sci. 99, 730-740 (2009).

24. Bakaletz, L.O., Kennedy, B.J., Novotny, L.A., Duquesne, G., Cohen, J. \& Lobet, Y. Protection against development of otitis media induced by nontypeable Haemophilus influenzae by both active and passive immunization in a chinchilla model of virus-bacterium superinfection. Infect. Immun. 67, 2746-2762 (1999).

25. Legge, K.L. \& Braciale, T.J. Accelerated migration of respiratory dendritic cells to the regional lymph nodes is limited to the early phase of pulmonary infection. Immunity 18, 265-277 (2003).

26. Novotny, L.A., Partida-Sanchez, S., Munson, R.S. Jr \& Bakaletz, L.O. Differential uptake and processing of a Haemophilus influenzae P5derived immunogen by chinchilla dendritic cells. Infect. Immun. 76, 967-977 (2008).

27. Geijtenbeek, T.B. et al. Identification of DC-SIGN, a novel dendritic cellspecific ICAM-3 receptor that supports primary immune responses. Cell 100, 575-585 (2000).

28. Valladeau, J. et al. Langerin, a novel C-type lectin specific to Langerhans cells, is an endocytic receptor that induces the formation of Birbeck granules. Immunity 12, 71-81 (2000).
29. Williams, R.L., Chalmers, T.C., Stange, K.C., Chalmers, F.T. \& Bowlin, S.J. Use of antibiotics in preventing recurrent acute otitis media and in treating otitis media with effusion. A meta-analytic attempt to resolve the brouhaha. JAMA 270, 1344-1351 (1993).

30. Prymula, R. et al. Pneumococcal capsular polysaccharides conjugated to protein $\mathrm{D}$ for prevention of acute otitis media caused by both Streptococcus pneumoniae and non-typeable Haemophilus influenzae: a randomised double-blind efficacy study. Lancet 367, 740-748 (2006).

31. Novotny, L.A., Jurcisek, J.A., Godfroid, F., Poolman, J.T., Denoel, P.A. \& Bakaletz, L.O. Passive immunization with human anti-protein D antibodies induced by polysaccharide protein $\mathrm{D}$ conjugates protects chinchillas against otitis media after intranasal challenge with Haemophilus influenzae. Vaccine 24, 4804-4811 (2006).

32. Koslap-Petraco, M.B. \& Parsons, T. Communicating the benefits of combination vaccines to parents and health care providers. J. Pediatr. Health Care 17, 53-57 (2003).

33. Jodar, L., Duclos, P., Milstien, J.B., Griffiths, E., Aguado, M.T. \& Clements, C.J. Ensuring vaccine safety in immunization programmes--a WHO perspective. Vaccine 19, 1594-1605 (2001).

34. O'Hagan, D.T. \& Rappuoli, R. Novel approaches to pediatric vaccine delivery. Adv. Drug. Deliv. Rev. 58, 29-51 (2006).

35. Holmgren, J. \& Czerkinsky, C. Mucosal immunity and vaccines. Nat. Med. 11 (4 Suppl), S45-S53 (2005).

36. Czerkinsky, C. \& Holmgren, J. Mucosal delivery routes for optimal immunization: targeting immunity to the right tissues. Curr. Top. Microbiol. Immunol. (2010); e-pub ahead of print 5 November 2010.

37. Male, C.J. Immunoglobulin A1 protease production by Haemophilus influenzae and Streptococcus pneumoniae. Infect. Immun. 26, 254-261 (1979).

38. Fernaays, M.M., Lesse, A.J., Cai, X. \& Murphy, T.F. Characterization of igaB, a second immunoglobulin $\mathrm{A} 1$ protease gene in nontypeable Haemophilus influenzae. Infect. Immun. 74, 5860-5870 (2006).

39. Kyd, J.M. \& Cripps, A.W. Modulation of antigen-specific T and B cell responses influence bacterial clearance of non-typeable Haemophilus influenzae from the lung in a rat model. Vaccine 14, 1471-1478 (1996)

40. Sirakova, T. et al. Role of fimbriae expressed by nontypeable Haemophilus influenzae in pathogenesis of and protection against otitis media and relatedness of the fimbrin subunit to outer membrane protein A. Infect. Immun. 62, 2002-2020 (1994).

41. Velin, D.M. \& Michetti, P. Advances in vaccination against Helicobacter pylori. Expert. Rev. Gastroenterol. Hepatol. 4, 157-166 (2010).

42. Okada, M.K. et al. Novel prophylactic and therapeutic vaccine against tuberculosis. Vaccine 27, 3267-3270 (2009).

43. Ha, S.W., West, E.E., Araki, K., Smith, K. \& Ahmed, R. Manipulating both inhibitory and stimulatory immune system towards the success of therapeutic vaccination against chronic viral infections. Immunol. Rev. 223, 317-333 (2008)

44. USDA licenses DNA vaccine for treatment of melanoma in dogs. J. Am. Vet. Med. Assoc. 236, 495 (2010).

45. Bowman, C.C. \& Clements, J.D. Differential biological and adjuvant activities of cholera toxin and Escherichia coli heat-labile enterotoxin hybrids. Infect. Immun. 69, 1528-1535 (2001).

46. Freytag, L.C. \& Clements, J.D. Mucosal adjuvants. Vaccine 23, 1804-1813 (2005)

47. Lawson, L.B., Clements, J.D. \& Freytag, L.C. Mucosal immune responses induced by transcutaneous vaccines. Curr. Top. Microbiol. Immunol. (2010); e-pub ahead of print 9 November 2010.

48. Zuercher, A.W. Upper respiratory tract immunity. Viral. Immunol. 16, 279-289 (2003).

49. Mathers, A.R. \& Larregina, A.T. Professional antigen-presenting cells of the skin. Immunol. Res. 36, 127-136 (2006).

50. Kissenpfennig, A. et al. Dynamics and function of Langerhans cells in vivo: dermal dendritic cells colonize lymph node areas distinct from slower migrating Langerhans cells. Immunity 22, 643-654 (2005).

51. Bal, S.M., Ding, Z., van Riet, E., Jiskoot, W. \& Bouwstra, J.A. Advances in transcutaneous vaccine delivery: do all ways lead to Rome? J. Control Release 148, 266-282 (2010).

52. Bakaletz, L.O., Tallan, B.M., Hoepf, T., DeMaria, T.F., Birck, H.G. \& Lim, D.J. Frequency of fimbriation of nontypable Haemophilus influenzae and its ability to adhere to chinchilla and human respiratory epithelium. Infect. Immun. 56, 331-335 (1988). 
53. Duim, B., Bowler, L.D., Eijk, P.P., Jansen, H.M., Dankert, J. \& van Alphen, L. Molecular variation in the major outer membrane protein P5 gene of nonencapsulated Haemophilus influenzae during chronic infections. Infect. Immun. 65, 1351-1356 (1997).

54. Webb, D.C. \& Cripps, A.W. Secondary structure and molecular analysis of interstrain variability in the P5 outer-membrane protein of non-typable Haemophilus influenzae isolated from diverse anatomical sites. J. Med. Microbiol. 47, 1059-1067 (1998).

55. Bakaletz, L.O., Leake, E.R., Billy, J.M. \& Kaumaya, P.T. Relative immunogenicity and efficacy of two synthetic chimeric peptides of fimbrin as vaccinogens against nasopharyngeal colonization by nontypeable Haemophilus influenzae in the chinchilla. Vaccine $\mathbf{1 5}$, 955-961 (1997)
56. Bakaletz, L.O. et al. Demonstration of type IV pilus expression and a twitching phenotype by Haemophilus influenzae. Infect. Immun. 73, 1635-1643 (2005).

57. Kyd, J.M., Cripps, A.W., Novotny, L.A. \& Bakaletz, L.O. Efficacy of the 26-kilodalton outer membrane protein and two P5 fimbrin-derived immunogens to induce clearance of nontypeable Haemophilus influenzae from the rat middle ear and lungs as well as from the chinchilla middle ear and nasopharynx. Infect. Immun. 71, 4691-4699 (2003).

(c) This work is licensed under the Creative Commons

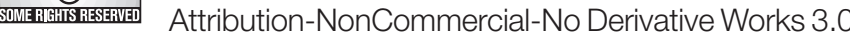
Unported License. To view a copy of this license, visit http://creativecommons.org/licenses/by-nc-nd/3.0/ 